\begin{tabular}{|c|c|c|}
\hline 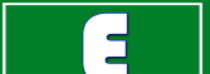 & International Journal of Current Research in & \\
\hline & Biosciences and Plant Biology & \\
\hline $\begin{array}{l}\text { EXCELLENT } \\
\text { PUBLISHERS } \\
\end{array}$ & $\begin{array}{c}\text { ISSN: 2349-8080 (Online) • Volume } 3 \bullet \text { Number } 1 \text { (January-2016) } \\
\text { Journal homepage: www.ijcrbp.com }\end{array}$ & \\
\hline
\end{tabular}

\title{
Reappraisal of Tribe Cariceae (Cyperaceae) in India
}

\author{
Masood Akhtar ${ }^{1}$ and R. C. Srivastava ${ }^{2 *}$ \\ ${ }^{1}$ Department of Botany, Shibli National College, Azamgarh (UP), India \\ ${ }^{2}$ Retired Scientist, Botanical Survey of India, Kolkata, India \\ *Corresponding author.
}

\begin{tabular}{|c|c|}
\hline Abstract & Article Info \\
\hline \multirow{19}{*}{$\begin{array}{l}\text { Sedge Genus Carex (Cyperaceae), with c. } 2000 \text { species, nearly cosmopolitan distribution } \\
\text { and broad range of habitats, is one of the largest angiosperm genera and the largest in } \\
\text { the temperate zone. Global Carex Group (2015) provided acceptable arguments and } \\
\text { evidences for a broader circumscription of Carex to add all species currently classified } \\
\text { in Cymophyllus (monotypic), Kobresia, Schoenoxiphium and Uncinia to those currently } \\
\text { classified as Carex. Carex and these genera comprise tribe Cariceae (subfamily } \\
\text { Cyperoideae, Cyperaceae) and form a well-supported monophyletic group in all } \\
\text { molecular phylogenetic studies to date. Carex as defined by GCG } 2015 \text { in the broad } \\
\text { sense comprises at least four clades. Three are strongly supported (Siderostictae, core } \\
\text { Vignea and core Carex), whereas the caricoid clade, which includes all the segregate } \\
\text { genera, receives only weak to moderate support. The caricoid clade is most commonly } \\
\text { split into two clades, one including a monophyletic Schoenoxiphium and two small } \\
\text { clades of species of Carex s.s., and the other comprising Kobresia, Uncinia and mostly } \\
\text { unispicate species of Carex s.s. Morphological variation is high in all but the Vignea } \\
\text { clade, making it extremely difficult to define consistent synapomorphies for most } \\
\text { clades. However, Carex s.l. as newly circumscribed by GCG 2015, is clearly } \\
\text { differentiated from the sister groups in tribe Scirpeae by the transition from bisexual } \\
\text { flowers with a bristle perianth in the sister group to unisexual flowers without a perianth } \\
\text { in Carex. The naked female flowers of Carex s.l. are at least partially enclosed in a } \\
\text { flask-shaped prophyll, termed a perigynium. Carex s.s. is not only by far the largest } \\
\text { genus in the group, but also the earliest published name. GCG } 2015 \text { proposed } 72 \text { new } \\
\text { combinations and } 58 \text { replacement names to treat all of tribe Cariceae as a single genus } \\
\text { Carex. The Indian scenario is presented in this communication following GCG } 2015 \\
\text { according to whom this broader monophyletic circumscription of Carex reflects the } \\
\text { close evolutionary relationships in the group and serves the goal of nomenclatural } \\
\text { stability better than other possible treatments. }\end{array}$} & $\begin{array}{l}\text { Accepted: } 28 \text { December } 2015 \\
\text { Available Online: O6 January } 2016\end{array}$ \\
\hline & Keywords \\
\hline & Cyperaceae \\
\hline & \\
\hline & Tribe Cariceae \\
\hline & \\
\hline & \\
\hline & \\
\hline & \\
\hline & \\
\hline & \\
\hline & \\
\hline & \\
\hline & \\
\hline & \\
\hline & \\
\hline & \\
\hline & \\
\hline & \\
\hline
\end{tabular}

\section{Introduction}

Much has been accomplished towards an understanding of Carex s.l. since the publication of Kükenthal's (1909) monograph, from species discovery to detailed analysis of floral and inflorescence morphology to DNA-based phylogenetic trees. Species of Carex s.l. continue to be discovered at a surprising rate even in well-explored areas, such as Europe, North America, Japan, Australia and New Zealand, and at even higher rates in China, South-East Asia, Africa and South America. Detailed studies of floral and inflorescence morphology have been conducted for most major groups of Carex s.l., although more work is needed on the Vigneastra group and 
Kobresia. At least one gene has been sequenced for more than half of the species in the tribe, and nuclear and plastid DNA regions, coding and non-coding, have been sequenced for more than half of these in ongoing phylogenetic studies. Despite this progress, there has been understandable hesitancy for anyone to reclassify a large and widespread group such as Cariceae unilaterally, despite frequent comments on the difficulty of distinguishing the genera in it. As a global group of Cyperaceae and Cariceae specialists, we think it is time to overcome the inertia of the traditional classification and make the required nomenclatural changes to recognize all species in the tribe as the genus Carex s.l. Much remains to be accomplished on a global scale to continue exploration and species discovery, to understand the ways in which genetic and environmental factors influence inflorescence variability within and between species, and to take advantage of the constantly expanding set of molecular tools and analytical methods to formulate phylogenetic hypotheses for the monophyletic genus Carex s.l.

The new broader circumscription of Carex proposed here is just the first step in the reclassification of the genus. The Global Carex Group is working towards a complete global sampling of species of Carex s.l., sequencing multiple DNA regions per species, to aid in placing species into natural sectional groups in the genus. Molecular phylogenetic work already completed on Carex section Ovales (Hipp et al., 2006), section Spirostachyae Drejer) L.H.Bailey (Escudero et al., 2007; Escudero and Luceño, 2009, 2011), section Phyllostachyae Tuck. ex Kük. (Starr et al., 1999) and section Ceratocystis Dumort. (Jiménez-Mejías, MartínBravo and Luceño, 2012; Derieg et al., 2013) illustrates the potential for clarifying the relationships at the sectional level with DNA analyses. Projects currently in progress by GCG include: an expanded phylogenetic analysis and new monograph of Schoenoxiphium as a section of Carex, a new molecular phylogenetic analysis of Carex subgenus Vignea, and additional work on the rest of the caricoid clade to elucidate natural groups in Kobresia and unispicate species. They are also working on reclassifications of sections Glareosae, Phleoideae (Meinsh.) T.V. Egorova and Remotae (Asch.) C.B.Clarke in the Vignea clade and sections Aulocystis Dumort., Chlorostachyae Tuckerm. ex Meinsh., Hymenochlaenae s.l., Porocystis, Phacocystis s.l., Racemosae, Mitratae Kük., Rhomboidales, Laxiflorae (Kunth) Mack., Paniceae G.Don, Bicolores, Careyanae Tuck. ex Kük., Griseae (L.H.Bailey) Kük., Granulares (O.Lang) Mack., Rostrales Meinsh., Vesicariae, Paludosae, Carex,
Lupulinae, Squarrosae J.Carey, Sylvaticae Rouy, Rhynchocystis Dumort. and Indicae Tuck. in the core Carex clade based on global sampling and informed by molecular data.

\section{Indian Kobresia species}

In accordance with this recent approach (GCG 2015), the Indian Kobresia species may now be referred as under:

1. Carex alatauensis Journal of the Linnean Society, 179, 1-42.2015. Elyna humilis C.A.Mey. ex Trautv., Trudy Imp. S.-Peterburgsk. Bot. Sada 1: 21. 187. 1871. Kobresia humilis (C.A.Mey. ex Trautv.) Serg. in Schischkin, Fl. URSS 3: 111. 1935. Kobresia royleana (Nees) Boeckeler var. humilis (C.A.Mey. ex Trautv.) Kük. in Engler (ed.), Pflanzenr. 38(IV, 20): 46. 1909; non Carex humilis Leyss. (1761). Kobresia persica Kük. \& Bornm., Oesterr. Bot. Z. 47: 133. 1897; non Carex persica Nelmes (1939). Kobresia royleana (Nees) Boeckeler var. parvinux T. Koyama, Acta Phytotax. Geobot. 16: 168. 1956.

Distribution: Central Asia, north-western China.

Etymology: The type of the Elyna humilis C.A.Mey. ex Trautv., was collected from the Alatau Mountains in Central Asia.

2. Carex bhutanensis S.R.Zhang, Journal of the Linnean Society,179, 1-42.2015. Kobresia prainii Kük., Bull. Herb. Boissier, sér. 2, 4: 50. 1904; non Carex prainii Kük. (1903). Kobresia utriculata C.B.Clarke, Bull. Misc. Inform. Kew, Addit. Ser. 8: 67. 1908; non Carex utriculata Boott (1939).Kobresia prainii Kük. var. elliptica Y.C.Yang in C.Y.Wu, Fl. Xizang. 5: 387, f. 217. 1987.

Distribution: Eastern Himalaya (Nepal, Sikkim,Bhutan) to south-western China.

Etymology: The type of the replaced name, Kobresia prainii Kük., was collected in Bhutan.

3. Carex bistaminata (W.Z.Di \& M.J.Zhong)S.R.Zhang. Journal of the Linnean Society, 179, 1-42. 2015. Kobresia bistaminata W.Z.Di \& M.J.Zhong, Acta Bot. Boreal.-Occid. Sin. 6(4): 275. 1986. Kobresia myosuroides (Vill.) Fiori ssp. bistaminata (W.Z.Di \& M.J.Zhong) S.R.Zhang, Novon, 9: 453. 1999.Kobresia kashgarica Dickoré, Stapfia, 39: 79. 1995.

Distribution: From Karakorum to western China. 
4. Carex bonatiana (Kük.) Ivanova, Bot. Zhurn.SSSR 24: 501. 1939. Kobresia bonatiana Kük., Bull. Géogr. Bot.22: 250. 1912.Kobresia fragilis C.B.Clarke, J. Linn. Soc., Bot. 36:267. 1903. Schoenoxiphium fragile (C.B.Clarke) C.B.Clarke, Bull. Misc. Inform. Kew, Addit. Ser. 8: 67.1908. non Carex fragilis Boott (1858). Carex curvata Boott, Ill. Gen. Carex 1: 2, pl. 5.1858, non Knaf (1847). Kobresia curvata C.B.Clarke,Bull. Misc. Inform. Kew, Addit. Ser. 8: 68. 1908.Schoenoxiphium clarkeanum Kük., Bull. Herb. Boissier, sér. 2, 4: 49. 1904. Kobresia clarkeana (Kük.)Kük. in Engler (ed.), Pflanzenr. 38(IV, 20): 48. 1909; non Carex clarkeana Kük. (1904). Kobresia clarkeana (Kük.) Kük. var. megalantha Kük., Bull. Géogr. Bot. 22: 249. 1912.Kobresia hispida Kük., Acta Horti Gothob. 5: 39.1930.Kobresia yuennanensis Hand.-Mazz., Symb. Sin. 7:1256. 1936. Kobresia curticeps (C.B.Clarke) Kük. var. gyirongensis Y.C.Yang in C.Y.Wu, Fl. Xizang. 5: 391, f. 222.1987.

Distribution: Nepal, India (Sikkim), Bhutan, southwestern China.

Note: The correct name for this species if the segregate genus is recognized is Kobresia fragilis.

5. Carex borealipolaris S.R.Zhang, Journal of the Linnean Society, 179, 1-42. 2015. Kobresia sibirica (Turcz. Ex Ledeb.) Boeckeler, Linnaea 39: 7. 1875. Elyna sibirica Turcz. ex Ledeb., Fl. Ross. 4(2): 262. 1852; non Carex sibirica Willd. ex Kunth (1837). Kobresia arctica A.E.Porsild, Sargentia 4: 15. 1943, non Meinsh. (1901).Kobresia macrocarpa Clokey ex Mack., N. Amer. Fl.18: 5. 1931. Kobresia bellardii (All.) Degl. ex Loisel.var. macrocarpa (Clokey ex Mack.) H.D.Harr., Man. Pl. Colorado 641. 1954; non Carex macrocarpa Phil.(1858).Kobresia smirnovii N.A.Ivanova, Bot. Zhurn.S.S.S.R. 24: 480. 1939; non Carex smirnovii V.I.Krecz.(1935).Kobresia hyperborea A.E.Porsild, Bull. Natl. Mus.Canada 121: 103. 1951; non Carex hyperborea Drejer (1841).Kobresia hyperborea A.E.Porsild var. alaskana Duman, Bull. Torrey Bot. Club 83: 194. 1956. Kobresia hyperborea A.E.Porsild var. lepagei Duman, Bull. Torrey Bot. Club 83: 194. 1956. Kobresia schoenoides (C.A.Mey.) Steud. var. lepagei (Duman) B.Boivin, Naturaliste Canad. 94: 525. 1967.

Distribution: Subarctic to north-western USA.

Etymology: The species is circumboreal, and common in the North Polar area. The epithet refers to the distributional pattern of the species.
6. Carex brandisii (C.B.Clarke ex Jana \& R.C.Srivast.) O.Yano, Journal of the Linnean Society, 179, 1-42. 2015. Kobresia brandisii C.B. Clarke ex Jana \& R.C.Srivast., J. Jap. Bot. 89: 205, f. 1 \& 2. 2014.

Distribution: Western Himalaya (India).

7. Carex breviprophylla O.Yano, Journal of the Linnean Society, 179, 1-42.2015. Kobresia gandakiensis Rajbh. \& H.Ohba in H. Ohba \& S. B. Malla (eds.), Himal. Pl. 2: 132, f. 4h-o. 1991; non Carex gandakiensis Katsuy. (2008).

Distribution: Nepal to India (Sikkim).

Etymology: This species is characterized by a prophyll that is shorter than the nutlet.

8. Carex capillifolia (Decne.) S.R.Zhang, Journal of the Linnean Society, 179, 1-42. 2015. Elyna capillifolia Decne. in Jacquemont,Voy. Inde. 4(Bot.): 173. 1844. Kobresia capillifolia (Decne.) C.B.Clarke, J. Linn. Soc., Bot. 20: 378. 1883. Elyna spicata Boiss., Fl. Orient. 5: 394. 1882, non Schrad. (1806). Kobresia brunnescens Boeckeler, Beitr. Cyper. 1: 40.1888.Kobresia elata Boeckeler, Beitr. Cyper. 2: 32. 1890. Kobresia macrolepis Meinsh., Trudy Imp.S.-Peterburgsk. Bot. Sada 18: 276. 1901. Elyna macrolepis(Meinsh.) Fomin \& Woronow, Opred. Rast.Kavk. 1: 173. 1909. Kobresia capilliformis Ivanova, Bot. Zhurn. SSSR 24: 484. 1939. Kobresia thomsonii Maxim. ex Ivanova, Bot. Zhurn.SSSR 24: 486. 1939, pro syn. Kobresia oviczinnikovii T.V.Egorova in Grubov, Pl.As. Centr. 3: 33. 1967, syn. nov. Kobresia yushuensis Y.C.Yang, Acta Biol. Plateau Sin. 2: 6. 1984.

Distribution: From Caucasus to western China.

9. Carex cercostachys Franch., Bull. Soc. Philom.Paris, 8, 7: 27. 1895. Kobresia cercostachys (Franch.) C.B.Clarke, J. Linn.Soc., Bot. 37: 267. 1903.Kobresia stiebritziana Hand.-Mazz., Akad. Wiss.Wien, Math.Naturwiss. Kl., Anz. 57: 54. 1920.Kobresia nepalensis (Nees) Kük. var. stiebritziana (Hand.-Mazz.) R.C.Srivast., Novon 8(2): 203. 1998.

Distribution: Bhutan, India (Sikkim), south-western China.

10. Carex clavispica S.R.Zhang, Journal of the Linnean Society,179, 1-42. 2015. Kobresia duthiei C.B.Clarke in J.D.Hooker, Fl. Brit. India 6: 697. 1894; non Carex 
duthiei C.B.Clarke (1894).Kobresia rostrata C.B.Clarke ex Ivanova, Bot.Zhurn. SSSR 24: 500. 1939, nom. nud., pro syn.; non Carex rostrata Stokes (1787).

Distribution: Himalaya, south-western China.

Etymology: The inflorescence of the species is a clavate spike.

11. Carex coninux (F.T.Wang \& Tang) S.R.Zhang, Journal of the Linnean Society, 179, 1-42. 2015. Kobresia coninux F.T.Wang \& Tang, Acta Phytotax. Sin. 1: 182. 1951. Kobresia pusilla Ivanova, Bot. Zhurn. SSSR 24:496. 1939; non Carex pusilla Arv.-Touv. (1872).Kobresia helanshanica W.Z.Di \& M.J.Zhong, Acta Bot. Boreal.-Occid. Sin. 5 (4): 311, f. 1. 1985. Kobresia daqingshanica X.Y.Mao, Acta Sci. Nat.Univ. Intramongol. 19(2): 341, f. 1. 1988. Kobresia arakorumensis Dickoré, Stapfia 39: 77.1995, syn. nov.

Distribution: Karakorum, Western Himalaya, northern and western China.

Note: The correct name for this species if the segregate genus is recognized is Kobresia pusilla.

12. Carex curticeps C.B.Clarke in J.D.Hooker, Fl.Brit. India 6: 749. 1894.Kobresia curticeps (C.B.Clarke) Kük. in Engler (ed.), Pflanzenr. 38(IV, 20): 47. 1909.

Distribution: Central \& eastern Himalaya to southern Tibet.

13. Carex deasyi (C.B.Clarke) O.Yano \& S.R.Zhang, Journal of the Linnean Society, 179, 1-42. 2015. Kobresia deasyi C.B.Clarke, Bull. Misc.Inform. Kew, Addit. Ser. 8: 68. 1908. Kobresia schoenoides (C.A.Mey.) Steud., Syn. Pl.Glumac. 2: 246. 1855. Elyna schoenoides C.A.Mey.,Verzeichn. Pfl. Cauc. Casp.: 29. 1831; non Carex schoenoides Schrank (1789).Kobresia pamiroalaica Ivanova, Bot. Zhurn. SSSR 24: 481. 1939.Kobresia pamiralaica Ivanova in R.R. Schreder, Fl.Uzbekist. 1: 347, 540. 1941.Kobresia septatonodosa Koyama, Acta Phytotax Geobot. 16: 168. 1956.Kobresia maquensis Y.C.Yang, Acta Biol. Plateau Sin. 2: 4, f. 3. 1984.Kobresia lacustris P.C.Li, Acta Bot. Yunnan. 12(1):14. 1990.Kobresia glaucifolia F.T.Wang \& Tang ex P.C.Li,Acta Phytotax. Sin. 37(2): 153. 1999.

Distribution: From Caucasus to western China.

Note: The correct name for this species if the segregate genus recognized is Kobresia schoenoides.
14. Carex esbirajbhandarii (Rajbh. \& H.Ohba)O.Yano, Journal of the Linnean Society,179, 1-42. 2015. Kobresia esbirajbhandarii Rajbh. \& H.Ohba, J. Jap. Bot. 62: 272. 1987.

Distribution: Nepal, (India?)

15. Carex esenbeckii Kunth, Enum. Pl. 2: 522. 1837. Kobresia esenbeckii (Kunth) Noltie, Edinburgh J. Bot. 50(1): 43. 1993. Kobresia esenbeckii (Kunth) F.T.Wang \& Tang ex P.C.Li in W.T.Wang, Vasc. Pl. Hengduan Mount. 2: 2352. 1994, later isonym. Carex trinervis Nees in Wight, Contr. Bot. India 120. 1834, non Degl. (1807). Kobresia trinervis (Nees)Boeckeler, Linnaea 39: 4. 1875. Hemicarex trinervis (Nees) C.B.Clarke, J. Linn. Soc., Bot. 20: 382. 1883.Kobresia seticulmis Boeckeler, Linnaea 39: 3. 1875.Holmia seticulmis (Boeckeler) Fedde \& J.Schust., Just's Bot. Jahresber. 41(2): 10. 1913 (publ. 1918).Kobresia hookeri Boeckeler, Linnaea 39: 4. 1875.Hemicarex hookeri (Boeckeler) Benth., J. Linn. Soc.,Bot. 18: 367. 1881.Carex mutans Boott ex C.B.Clarke, J. Linn. Soc.,Bot. 20: 383. 1883.Carex polygyna Boeckeler, Beitr. Cyper. 1: 40. 1888.Kobresia hookeri Boeckeler var. dioica C.B.Clarke in J.D.Hooker, Fl. Brit. India 6: 695. 1894.Kobresia angusta C.B.Clarke in J.D.Hooker, Fl.Brit. India 6: 695. 1894.Kobresia foliosa C.B.Clarke in J.D.Hooker, Fl. Brit.India 6: 696. 1894.Kobresia trinervis (Nees) Boeckeler var. foliosa(C.B.Clarke) Kük. in Engler (ed.), Pflanzenr. 38(IV, 20): 43. 1909.

Distribution: Himalaya to south-western China.

16. Carex filispica S.R.Zhang, Journal of the Linnean Society, 179, 1-42.2015. Hemicarex filicina C.B.Clarke, J.Linn. Soc., Bot. 20: 384. 1883. Kobresia filicina (C.B.Clarke) C.B.Clarke in J.D.Hooker, Fl. Brit. India 6: 696. 1894; non Carex filicina Nees (1834).Kobresia filicina (C.B.Clarke) C.B.Clarke var. subfilicinoides P.C.Li, Acta Phytotax. Sin. 37(2): 155.1999; non Carex subfilicinoides Kük. (1930).

Distribution: Eastern Himalaya (India), south-western China.

Etymology: The first part of the name, fili-, threadlike,from filum, a thread, here refers to the narrowly linear spike of this species.

17. Carex fissiglumis (C.B.Clarke) S.R.Zhang \& O.Yano Journal of the Linnean Society, 179, 1-42. 2015. Kobresia fissiglumis C.B.Clarke in J.D.Hooker, Fl. Brit. 
India 6: 696. 1894. Kobresia esenbeckii (Kunth) Noltie var. fissiglumis (C.B.Clarke) Noltie, Edinburgh J. Bot. 50(1): 43. 1993.

Distribution: Central Himalaya (western Nepal) to southwestern China.

18. Carex gammiei (C.B.Clarke) S.R.Zhang \& O.Yano, Journal of the Linnean Society, 179, 1-42. 2015. Kobresia gammiei C.B.Clarke, Bull. Misc. Inform. Kew, Addit. Ser. 8: 68. 1908. Kobresia williamsii Koyama, Bot. Mag. (Tokyo) 86(1004): 279, pl. 3. 1973.

Distribution: Nepal, Bhutan, India (Sikkim), southwestern China.

19. Carex handel-mazzettii (Ivanova) S.R.Zhang, Journal of the Linnean Society, 179, 1-42. 2015. Kobresia handel-mazzettii Ivanova, Bot. Zhurn. SSSR 24: 494. 1939. Kobresia capillifolia (Decne.) C.B.Clarke var. condensata Kük., Notes Roy. Bot. Gard. Edinburgh 7: 134. 1912. Kobresia condensata (Kük.) S.R.Zhang \& Noltie, Fl.China 23: 274. 2010. Kobresia royleana (Nees) Boeckeler var. himalaica Rajbh. \& H.Ohba in H. Ohba \& S. B. Malla (eds.),Himal. Pl. 2: 150. 1991.

Distribution: South-western China (Sichuan, Tibet, Yunnan), Nepal, India (?)

20. Carex harae (Rajbh. \& H.Ohba) O.Yano, Journal of the Linnean Society, 179, 1-42. 2015. Kobresia harae Rajbh. \& H.Ohba, J. Jap. Bot. 62: 193 (1987), as 'harai'.

Distribution: India, Nepal.

21. Carex hughii S.R.Zhang, Journal of the Linnean Society, 179, 1-42. 2015. Kobresia graminifolia C.B.Clarke, J. Linn. Soc., Bot. 36: 268. 1903; non Carex graminifolia Cherm. (1923).

Distribution: Western China, India (?)

Etymology: The epithet of the species is adopted to commemorate Rev. Fr. Hugh, the collector of the type of Kobresia graminifolia C.B.Clarke.

22. Carex kanaii (Rajbh. \& H.Ohba) S.R.Zhang \& O.Yano. Journal of the Linnean Society, 179, 1-42.2015. Kobresia kanaii Rajbh. \& H.Ohba in H. Ohba \& S. B. Malla (eds.), Himal. Pl. 2: 135, f. 6. 1991.

Distribution: Nepal, Sikkim.
23. Carex kokanica (Regel) S.R. Zhang Journal of the Linnean Society, 179, 1-42. 2015. Elyna kokanica Regel, Trudy Imp. S.-Peterburgsk. Bot. Sada 7: 563. 1880. Trilepis royleana Nees, Linnaea 9: 305. 1834; non Carex royleana Nees (1834). Kobresia royleana (Nees) Boeckeler, Linnaea 39: 8. 1875. Kobresia stenocarpa (Kar. \& Kir.) Steud. var royleana (Nees) C.B.Clarke, J. Linn. Soc., Bot. 20: 381. 1883.Elyna stenocarpa Kar. \& Kir., Bull. Soc. Imp. Naturalistes Moscou 15(3): 526. 1842; non Carex stenocarpa Turcz. ex Krecz. (1935). Kobresia stenocarpa (Kar. \& Kir.) Steud., Synop. Pl. Glum. 2: 246. 1854.Kobresia stenocarpa (Kar. \& Kir.) Steud. var.simplex Y.C.Yang in C.Y.Wu, Fl. Xizang. 5: 395, f. 224. 1987. Kobresia paniculata Meinsh., Trudy Imp.S.-Peterburgsk. Bot. Sada 18(3): 279. 1901. Kobresia royleana (Nees) Boeckeler var. paniculata (Meinsh.) Kük. in Engler (ed.), Pflanzenr. 38(IV, 20): 46. 1909.K. minshanica F.T.Wang \& Tang ex Y.C.Yang, Acta Biol. Plateau Sin. 2: 1. 1984. Kobresia royleana (Nees)Boeckeler ssp. minshanica (F.T.Wang \& Tang ex Y.C.Yang) S.R.Zhang, Novon 9: 453. 1999. K. menyuanica Y.C.Yang, Acta Biol. Plateau Sin. 2:3. 1984.

Distribution: Western Asia (Afghanistan), Central Asia, Himalaya, western China.

Note: The correct name for this species if the segregate genus is recognized is Kobresia royleana.

24. Carex macroprophylla (Y.C.Yang) S.R.Zhang, Journal of the Linnean Society, 179, 1-42. 2015. Kobresia filifolia (Turcz.) C.B.Clarke var.macroprophylla Y.C.Yang, Acta Biol. Plateau Sin. 2: 8,f. 5. 1984. Kobresia macroprophylla (Y.C.Yang) P.C.Li in L.K.Dai \& S.Y.Liang, Fl. Reipubl. Popul. Sin. 12:17. 2000.Kobresia filifolia (Turcz.) C.B.Clarke, J. Linn. Soc.,Bot. 20: 381. 1883. Elyna filifolia Turcz., Bull. Soc.Imp. Naturalistes Moscou 28(1): 353. 1855; non Carex filifolia Nutt. (1818). Kobresia capillifolia (Decne.)C.B.Clarke var. filifolia (Turcz.) Kük., Finska Vet.-Soc. Föhr. 65(8): 1. 1902-1903.Kobresia gracilis Meinsh., Acta Horti Petrop. 18:276. 1901; non Carex gracilis Curtis (1782).Kobresia pratensis Freyn, Oesterr. Bot. Z. 40: 266.1890; non Carex pratensis Hosé (1797).

Distribution: Northern China, Mongolia, Siberia.

Note: The correct name for this species if the segregate genus is recognized is Kobresia filifolia.

25. Carex myosuroides Vill., Prosp. Hist. Pl.Dauphine 1718. 1779. Kobresia myosuroides (Vill.) Fiori in Fiori et al., 
Fl.Anal. Ital. 1: 125. 1896. Elyna myosuroides (Vill.)Fritsch ex Janch., Mitt. Naturwiss. Vereins Univ.Wien 5: 110. 1907.Carex bellardii All., Fl. Pedem. 2: 264. 1785. Kobresia bellardii (All.) Degl. ex Loisel., Fl. Gall. 2: 626.1807.Carex hermaphrodita J. F. Gmel, Syst. Nat. 2: 138.1791.Kobresia scirpina Willd., Sp. Pl. ed. 4, 4(1): 205. 1805. Elyna spicata Schrad., Fl. Germ. 1: 155. 1806.Carex affinis R.Br., Bot. App. 750. 1823. Kobresia nardina Hornem. in G. C. Oeder \& al., Fl.Dan. 74. 1827.Kobresia filiformis Dewey, Amer. J. Sci. Arts 29:253. 1836. Elyna filiformis Steud., Syn. Pl. Glumac. 2: 245.1855.Carex vulcanicola Nakai, Bot. Mag. (Tokyo) 28: 327.1914.

Distribution: Europe, northern Asia, northern North America, Greenland.

26. Carex neesii S.R.Zhang, Journal of the Linnean Society,179, 1-42.2015. Kobresia nepalensis (Nees) Kük. in Engler (ed.), Pflanzenr. 38(IV, 20): 46: 40, f. 9. 1909. Uncinia nepalensis Nees in Wight, Contr. Bot. India 129. 1834; non Carex nepalensis Spreng. (1826).Carex linearis Boott, Ill. Gen. Carex 1: 51, pl. 136.1858, non Clairv. (1811).

Distribution: Western Himalaya to south-western China.

Etymology: The epithet of the species is adopted to commemorate Christian Gottfried Daniel Nees von Esenbeck (1776-1858) who published the first name for this species.

27. Carex elachista (C.B.Clarke) R.C.Srivast, comb.et status nova. Kobresia nepalensis (Nees) Kük. var elachista (C.B.Clarke) Kük. in Engler (ed.), Pflanzenr. 38(IV, 20): 40. 1909. Carex linearis Boott var. elachista C.B.Clarke in J.D.Hooker, Fl. Brit. India 6: 713. 1894.

Distribution: India, Nepal.

28. Carex nudicarpa (Y.C.Yang) S.R.Zhang, Journal of the Linnean Society, 179, 1-42. 2015. Blysmocarex nudicarpa Y.C.Yang, Acta Bot. Yunnan. 4(4): 325. 1982. Kobresia nudicarpa(Y.C.Yang) S.R.Zhang, Acta Phytotax. Sin. 33(2): 160.1995. Kobresia macrantha Boeckeler var. nudicarpa (Y.C.Yang) P.C.Li in L.K.Dai \& S.Y.Liang, Fl. Reipubl.Popul. Sin. 12: 26. 2000. Blysmocarex macrantha (Boeckeler) Ivanova ssp. nudicarpa (Y.C.Yang)D.S.Deng, Guihaia 22: 120. 2002. Kobresia macrantha Boeckeler, Beitr. Cyper. 1: 39.1888. Blysmocarex macrantha (Boeckeler) Ivanova, Bot. Zhurn. SSSR 24: 502. 1939; non Carex macrantha Boeckeler (1888).
Distribution: South-western China, Himalaya eastern Karakorum.

Note: The correct name for this species if the segregate genus is recognized is Kobresia macrantha.

29. Carex ovoidispica O.Yano, Journal of the Linnean Society, 179, 1-42. 2015. Kobresia nitens C.B.Clarke, J.Linn. Soc. Bot. 20: 379, pl. 30, f. 7. 1883; non Carex nitens Phil. (1873).

Distribution: India, Pakistan to Nepal.

Etymology: The specific epithet refers to the conspicuous ovoid spikes.

30. Carex paramjitii (Jana, Noltie, R.C.Srivast \& A.Mukh.) O.Yano, Journal of the Linnean Society, 179, 1-42. 2015. Kobresia paramjitii Jana, Noltie, R.C.Srivast \& A.Mukh., Indian J. Plant Sci. 3 (online): 106.2014.

Distribution: India (Sikkim).

31. Carex parvula O.Yano, Journal of the Linnean Society, 179, 1-42. 2015. Kobresia pygmaea (C.B.Clarke) C.B.Clarke in J.D.Hooker, Fl. Brit. India 6: 696. 1894. Hemicarex pygmaea C.B.Clarke, J. Linn. Soc., Bot.20: 383. 1883; non Carex pygmaea Boeckeler (1876). Kobresia pygmaea C.B.Clarke var. filiculmis Kük.,Acta Horti Gothob. 5: 37. 1930; non Carex filiculmis Franch. \& Sav. (1878). Kobresia microstachya Ivanova, Bot. Zhurn. SSSR 24: 488. 1939; non Carex microstachya Ehrh. (1784).Kobresia koelzii Kük. ex Ivanova, Bot. Zhurn. SSSR 24: 498. 1939, pro syn.

Distribution: From Himalaya (India) to northern and western China.

Etymology: The specific epithet refers to the dwarf habit.

32. Carex prainii Kük., Bull. Herb. Boissier, sér. 2, 4:51. 1903. Kobresia sikkimensis Kük. in Engler (ed.), Pflanzenr. 38 (IV, 20): 47. 1909; non Carex sikkimensis C.B. Clarke (1894).

Distribution: Nepal to India (Assam).

33. Carex pseudogammiei S.R.Zhang, Journal of the Linnean Society, 179, 1-42. 2015. Kobresia loliacea F.T.Wang \&Tang ex P.C.Li, Acta Bot. Yunnan. 12(1): 13. 1990; non Carex loliacea L. (1753). Distribution: South-western China (north-western Yunnan, western Sichuan). 
Etymology: The species is morphologically similar to Carex gammiei (C.B.Clarke) S.R.Zhang.

34. Carex pseudolaxa (C.B.Clarke) O.Yano \& S.R.Zhang, Journal of the Linnean Society, 179, 142.2015. Kobresia pseudolaxa C.B.Clarke, J. Linn Soc., Bot. 20: 381. 1883.Kobresia laxa Nees in Wight, Contr. Bot. India 119.1834; non Carex laxa Wahlenb. (1803). Elyna laxa(Nees) Kunth, Enum. Pl. 2: 534. 1837. Hemicarex laxa(Nees) Benth., J. Linn. Soc., Bot. 18: 367. 1881. Schoenoxiphiumlaxum (Nees) Ivanova, Bot. Zhurn. SSSR 24: 501. 1939. Schoenoxiphium hissaricum Pissjauk., Bot. Mater.Gerb. Bot. Inst. Komarova Akad. Nauk SSSR. 12: 72.1950. Kobresia hissarica (Pissjauk.) Soják, Nár. Mus.Odd. Prír. 148: 194. 1979 (publ. 1980). Kobresia laxa Nees ssp. hissarica (Pissjauk.) Kukkonen, Ann.Naturhist. Mus. Wien, B 98B (Suppl.): 91. 1996. Kobresia afghanica Raymond, Dansk Bot. Ark. 14: 17. 1965.

Distribution: India (Kashmir) to central Himalaya.

Note: The correct name for this species if the segregate genus is recognized is Kobresia laxa.

35. Carex pseuduncinoides (Noltie) O.Yano \& S.R.Zhang, Journal of the Linnean Society, 179, 1-42. 2015. Kobresia pseuduncinoides Noltie, Edinburgh J. Bot. 50(1): 47, f. 1a-g. 1993.Kobresia kansuensis Kük., Acta Horti Gothob. 5: 38.1930; non Carex kansuensis Nelmes (1939).

Distribution: Bhutan, India, Nepal, western China.

Note: The correct name for this species if the segregate genus is recognized is Kobresia kansuensis.

36. Carex resrivastavae (Jana) E.H.Roalson, Journal of the Linnean Society, 179, 1-42. 2015. Kobresia rcsrivastavae Jana, Indian J.Fundam. Appl. Life Sci. 2: 256. 2012.

Distribution: India (Uttarakhand).

37. Carex sanguinea Boott, Proc. Linn. Soc. London 1: 285. 1846. Kobresia sanguinea (Boott) Raymond, Biol. Skr. 14(4):19. 1965.

Distribution: Eastern Afghanistan to western Himalaya.

38. Carex sargentiana (Hemsl.) S.R.Zhang, Journal of the Linnean Society, 179, 1-42. 2015. Kobresia sargentiana Hemsl., J. Linn.Soc., Bot. 30: 139. 1894. Kobresia robusta Maxim. var.sargentiana (Hemsl.) Kük. in Engler (ed.), Pflanzenr.38(IV, 20): 36. 1909.Kobresia robusta Maxim., Bull. Acad. Imp. Sci. St.-Petersbourg, n.s., 29: 218. 1883; non Carex robusta F.Nyl. (1844).

Distribution: Western China, India (Sikkim).

Note: The correct name for this species if the segregate genus is recognized is Kobresia robusta.

Distribution: India, China

39. Carex siamensis (Ohwi) S.R.Zhang, Journal of the Linnean Society, 179, 1-42. 2015. Kobresia siamensis Ohwi, Acta Phytotax.Geobot. 23: 109. 1968. Kobresia curvirostris (C.B.Clarke) C.B.Clarke in J.D.Hooker, Fl. Brit. India 6: 699. 1894. Hemicarex curvirostris C.B.Clarke, J. Linn. Soc., Bot. 20: 384.1883; non Carex curvirostra Hartm. (1832).

Distribution: Eastern Himalaya to northern Thailand.

Note: The correct name for this species if the segregate genus is recognized is Kobresia curvirostris.

40. Carex simpliciuscula Wahlenb., Kongl. Vetensk. Acad. Nya Handl. 24(2): 141. 1803. Kobresia simpliciuscula (Wahlenb.) Mack., Bull.Torrey Bot. Club 50: 349. 1923.Carex bipartita All., Fl. Pedem. 2: 265. 1785, nom.rej. Kobresia bipartita (All.) Dalla Torre, Atlas Alpenfl. 2: 216. 1882. Schoenus monoicus Sm., Engl. Bot. t. 1410. 1805. Kobresia caricina Willd., Sp. Pl. ed. 4, 4: 206. 1805. Carex lacustris Balbis ex Willd., Sp. Pl. ed. 4, 4:206. 1805, pro syn.Carex hybrida Schuhr ex Willd., Sp. Pl. 4: 206.1805, pro syn.Carex mirabilis Host, Icon. Descr. Gram. Austriac.4: 44, pl. 78. 1809. Elyna caricina Mert. et Koch in Röhling, Deutschl.Fl. ed. 3, 1: 458. 1823. Carex lobata Willd. ex Kunth, Enum. Pl. 2: 533.1837, pro syn.Kobresia simpliciuscula (Wahlenb.) Mack. var. americana Duman, Bull. Torrey Bot. Club 83: 194.1956.Kobresia filifolia (Turcz.) C.B.Clarke ssp. subfilifolia T.V.Egorova, Jurtzev \& V.V.Petrovsky, Bot. Zhurn.(Moscow \& Leningrad) 66(7): 1042. 1981. Kobresia simpliciuscula (Wahlenb.) Mack. ssp. subfilifolia (T.V.Egorova, Jurtzev \& V.V.Petrovsky) T.V.Egorova,Novosti Sist. Vyssh. Rast., 20: 84. 1983. Kobresia simpliciuscula (Wahlenb.) Mack. var. subfilifolia (T.V.Egorova, Jurtzev \& V.V.Petrovsky) A.E.Kozhevn.,Sosud. Rast. Sovet. Dal'nego Vostoka, 3: 229. 1988. Kobresia simpliciuscula (Wahlenb.) Mack. ssp. Subholarctica T.V.Egorova, Novosti. Sist. Vyssh. Rast., 20:83. 1983. Kobresia simpliciuscula (Wahlenb.) 
Mack.var. subholarctica (T.V.Egorova) A.E.Kozhevn., Sosud.Rast. Sovet. Dal'nego Vostoka, 3: 229. 1988. Kobresia subholarctica (T.V.Egorova) T.V.Egorova, Bot. Zhurn. (Moscow \& Leningrad) 76(12): 1736 1991.

Distribution: Europe to Caucasus, subarctic America to western USA.

41. Carex squamiformis (Y.C.Yang) S.R.Zhang, Journal of the Linnean Society, 179, 1-42. 2015. Kobresia squamiformis Y.C.Yang, Acta Biol. Plateau Sin. 2: 9, f. 6. 1984, published as: 'squmaeformis'. Kobresia setschwanensis Hand.-Mazz. ssp. squamiformis (Y.C.Yang) S.R.Zhang, Novon 9: 453. 1999.

Distribution: India, China.

42. Carex tibetikobresia S.R.Zhang, Journal of the Linnean Society, 179, 1-42. 2015. Kobresia tibetica Maxim., Bull.Acad. Imp. Sci. St.-Petersbourg, n.s., 29: 219. 1884. Kobresia capillifolia (Decne.) C.B.Clarke var.tibetica (Maxim.) Kük. in Engler (ed.), Pflanzenr.38(IV, 20): 36. 1909; non Carex thibetica Franch.,orth. var. (1887).

Distribution: Bhutan, India, western China.

Etymology: The epithet is based on the replaced synonym, Kobresia tibetica. The species is mainly distributed in the eastern Tibetan Plateau.

43. Carex uncinoides Boott, Ill. Gen. Carex 1: 8, pl.23. 1858. Kobresia uncinoides (Boott) C.B.Clarke in J.D.Hooker, Fl. Brit. India 6: 698. 1894.

Distribution: Eastern Himalaya (Nepal to Bhutan), northern Myanmar, south-western China.

44. Carex vaginosa (Jana, R.C.Srivast \& Bhaumik)O.Yano, Journal of the Linnean Society, 179, 1-42. 2015. Kobresia vaginosa C.B.Clarke in J.D.Hooker, Fl. Brit. India 6: 695. 1894. Kobresia nepalensis (Nees) Kük. var. vaginosa (C.B.Clarke)Kük. in Engler, Pflanzenr. 38(IV, 20): 40. 1909. Kobresia nepalensis (Nees) Kük. ssp. vaginosa (C.B.Clarke) Koyama in Hara et al., Enum. Fl. Pl.Nepal 1: 113. 1978. Kobresia nepalensis (Nees) Kük.var. vaginosa (C.B.Clarke) R.C. Srivast., Fl. Sikkim 1:225. 1996. Kobresia cercostachys (Franch.) C.B.Clarke var.capillacea P.C.Li, Acta Bot. Yunnan. 12(1): 17. 1990.
Distribution: Nepal, India (Sikkim), south-western China.

45. Carex vibhae (Jana, R.C.Srivast \& Bhaumik) O.Yano, Journal of the Linnean Society, 179, 1-42. 2015. Kobresia vibhae Jana, R.C.Srivast \& Bhaumik, Indian J. Plant Sci. 3 (online): 110, pl. 5.2014.

Distribution: South-eastern Himalaya.India (Arunachal Pradesh)

46. Carex vidua Boott ex C.B.Clarke in J.D.Hooker, Fl.Brit. India 6: 713. 1894.Kobresia vidua (Boott ex C.B.Clarke) Kük. in Engler (ed.), Pflanzenr. 38(IV, 20): 40. 1909. Kobresia prattii C.B.Clarke, J. Linn. Soc., Bot. 36:268. 1903.

46. Kobresia harrysmithii Kük., Acta Horti Gothob. 5:37. 1930 .

Distribution: Himalaya [Nepal, India (Sikkim), Bhutan] to western China.

\section{Conflict of interest statement}

Authors declare that they have no conflict of interest.

\section{Acknowledgement}

The Authors gratefully acknowledge the Botanical Journal of the Linnean Society and The Linnean Society of London.

\section{References}

Derieg, N.J., Weil, S.J., Reznicek, A.A., Bruederle, L.P., 2013. Carex viridistellata sp. nov. (Cyperaceae), a new cryptic species from prairie fens of the eastern United States. Syst. Bot. 38, 82-91.

Escudero, M., Luceño, M., 2009. Systematics and evolution of Carex sects. Spirostachyae and Elatae (Cyperaceae). Plant Syst. Evol. 279, 163-189.

Escudero, M., Luceño, M., 2011. Taxonomic revision of the tropical African group of Carex subsect. Elatae (sect. Spirostachyae, Cyperaceae). Anales del Jardin Bot. Madrid 68, 225-247.

Escudero, M., Valcárcel, V., Vargas, P., Luceño, M., 2007. Evolution in Carex L. sect. Spirostachyae (Cyperaceae): a molecular and cytogenetic approach. Organ. Diver. Evol. 7, 271-291.

GCG (Global Carex Group), 2015. Making Carex monophyletic (Cyperaceae, tribe Cariceae): a new broader circumscription. Bot. J. Linn. Soc. 179, 1-42. 
Hipp, A.L., Reznicek, A.A., Rothrock, P.E., Weber, J.A., 2006. Phylogeny and classification of Carex section Ovales (Cyperaceae). Int. J. Plant Sci. 167(5):1029-1048.

Jana, B., Srivastava, R.C., 2014. Genus Kobresia Willd. (Cyperaceae) in India. Indian J. Pl. Sci. 3, 91-111.

Jiménez-Mejías, P., Martín-Bravo, S., Luceño, M., 2012. Systematics and taxonomy of Carex sect. Ceratocystis (Cyperaceae) in Europe: a molecular and cytogenetic approach. Syst. Bot. 37, 382-398.
Kükenthal, G., 1909. Cyperaceae-Caricoideae. In: Das Pflanzenreich IV. 20, Heft 38. Engelmann, Leipzig. pp. 1824.

Starr, J.R., Bayer, R.J., Ford, B.A., 1999. The phylogenetic position of Carex section Phyllostachys and its implications for phylogeny and subgeneric circumscription in Carex (Cyperaceae). Am. J. Bot. 86, 563-577.

\section{How to cite this article:}

Akhtar, M., Srivastava, R.C., 2016. Reappraisal of Tribe Cariceae (Cyperaceae) in India. Int. J. Curr. Res. Biosci. Plant Biol. 3(1), 104-112. doi: http://dx.doi.org/10.20546/ijcrbp.2016.301.011 